\title{
Characterization of cerebral blood flow dynamics with multiscale entropy
}

\author{
Alexey N. Pavlov* \\ Yuri Gagarin State Technical University of Saratov \\ 77 Politechnicheskaya Street, Saratov 410054, Russia \\ pavlov.alexeyn@gmail.com \\ Arkady S. Abdurashitov, Olga N. Pavlova, Maria V. Ulanova, \\ Anastasia A. Bodrova, Natalia A. Shushunova, \\ Alexander P. Khorovodov and Oxana V. Semyachkina-Glushkovskaya ${ }^{\dagger}$ \\ Saratov National Research State University \\ 83 Astrakhanskaya Street, Saratov 410012, Russia \\ ${ }_{\dagger}^{\dagger}$ lushkovskaya@mail.ru \\ Received 19 June 2017 \\ Accepted 15 August 2017 \\ Published 12 September 2017

\begin{abstract}
Based on the laser speckle contrast imaging (LSCI) and the multiscale entropy (MSE), we study in this work the blood flow dynamics at the levels of cerebral veins and the surrounding network of microcerebral vessels. We discuss how the phenylephrine-related acute peripheral hypertension is reflected in the cerebral circulation and show that the observed changes are scale-dependent, and they are significantly more pronounced in microcerebral vessels, while the macrocerebral dynamics does not demonstrate authentic inter-group distinctions. We also consider the permeability of blood-brain barrier (BBB) and study its opening caused by sound exposure. We show that alterations associated with the BBB opening can be revealed by the analysis of blood flow at the level of macrocerebral vessels.
\end{abstract}

Keywords: Cerebral blood flow; laser speckle contrast imaging; arterial pressure; complexity.

\section{Introduction}

Complexity measures ${ }^{1-4}$ are related to informative characteristics reflecting the functioning of biological systems. Normal physiological dynamics is typically associated with a high degree of complexity caused by the influences of different regulatory mechanisms. A loss of this complexity is a sign of possible distortions in the mechanisms of normal physiological regulation and the development of diseases. ${ }^{5,6}$ On the contrast, extreme randomness of the dynamics may reflect a disbalance in the

${ }^{*}$ Corresponding author.

This is an Open Access article published by World Scientific Publishing Company. It is distributed under the terms of the Creative Commons Attribution 4.0 (CC-BY) License. Further distribution of this work is permitted, provided the original work is properly cited. 


\section{A. N. Pavlov et al.}

regulation indicating another type of the transformation of normal functioning regime into a pathological one. Therefore, a healthy dynamics should correspond to some "intermediate" degree of complexity, and its quantification is important for monitoring of the organism's state. Elaboration of diagnostic markers being able to separate normal and pathological dynamics assumes establishing of pronounced distinctions between the corresponding physiological signals. Taking into account the multiscale structure of such signals, ${ }^{7,8}$ quantification of the expected distinctions should be provided depending on the scale. When the complexity of a system is characterized with a single quantity, the resulting measure is less appropriate and informative. Thus, a reduction of complexity of beat-tobeat intervals may be a sign of disease; however, it does not provide enough information for understanding a reason of such reduction, and mechanism responsible for the corresponding changes generally remain unclear. A mechanism-related characterization of the complexity is a more perspective topic. ${ }^{9}$

This tendency can be traced in recent studies of physiological processes with entropy-based approaches. Entropy is often considered as a complexity measure that is able to characterize the dynamics of biological systems. Several entropy-based analyses were applied in physiological studies, including Shannon entropy, ${ }^{1}$ Kolmogorov-Sinai entropy, approximate entropy (ApEn), ${ }^{3}$ sample entropy (SampEn), ${ }^{4}$ etc. ApEn characterizes the irregularity or the randomness of time series depending on two parameters, pattern length and tolerance factor. ${ }^{10}$ This measure is easily applied to physiological data; however, it is not appropriate when dealing with short and noisy time series. In order to improve some shortcomings of ApEn (such as the requirement of large data set and inconsistent results), a modified measure, the SampEn was proposed. ${ }^{3}$ Advantages of this quantity over the ApEn are discussed in several studies. ${ }^{3,11}$ Aiming to describe the complexity of physiological time series as a function of the time scale, the multiscale entropy (MSE) was developed which is based on SampEn. ${ }^{12-14}$ This complexity measure has found many applications in physiological studies because it is adapted to characterize hierarchical complexity of biological processes.

In this work, we apply the MSE method to study blood flow dynamics at the levels of macro and microcerebral circulation. In order to analyze cerebral blood flow (CBF) noninvasively, we use laser speckle contract imaging (LSCI) being the optical coherent-domain method that provides a high spatio-temporal resolution. ${ }^{15,16}$ Scattering of the coherent light from moving particles of the blood produce the speckle pattern whose temporal changes allow describing CBF. ${ }^{17}$ LSCI is a widely applied method for functional visualization in biology and medicine ${ }^{16}$ even in high scattering tissues. This method can provide qualitative blood flow images within the full field of view without any scanning hardware. Minimum hardware requirements and relatively simple processing algorithm make LSCI an ideal tool for our research. The main drawback of such technique is a rather complicated interpretation process in order to recover the absolute flow speed. In experimental studies, LSCI is popular for the monitoring of $\mathrm{CBF}$ and oxygen saturation of the brain tissues. ${ }^{18}$ LSCI is a promising method to shed new light on the nature of vascular catastrophes in the brain. ${ }^{19,20} \mathrm{We}$ have recently developed an approach for LSCI separate analysis of arterial and venous responses to stress. ${ }^{21,22}$ In our experiments on rats with stressinduced intracranial hemorrhages, we showed that cerebral veins are more sensitive to harmful effects of stress compared with cerebral arteries and microvessels. LSCI experimental data allowed us to understand that accumulation of extensive blood in venous network is a crucial factor for stress-induced critical changes in cerebral vessels related to intracranial hemorrhages. ${ }^{23-25}$ With LSCI, we also found that the cerebral veins are more sensitive to adrenergic stimulation under normal state and during stress. Full-field imaging of cerebral vessels in rats represents an important new insight in our understanding how the brain responds to stress: after stress-induced intracranial hemorrhages, rats demonstrate a typical venous relaxation due to activation of a special type of vascular receptors "anarchists" that are stimulated by adrenaline the main hormone of stress but not nervous system. ${ }^{17}$ This fact explains why hyperperfusion is often associated with stress-related stroke in rodents. Our previous LSCI data were the reasons to choose this method for further more detailed study of adrenergic system in regulation of $\mathrm{CBF}$ focusing on the analysis of the effects of high blood pressure on the $\mathrm{CBF}$ as a main reason for the stroke.

Using LSCI, we consider here the responses of the $\mathrm{CBF}$-dynamics to the acute peripheral hypertension 
in rats and study alterations associated with the opening of blood-brain barrier (BBB) in mice. The paper is organized as follows. In Sec. 2, we describe experimental methods and data processing tools. A study of typical changes of CBF-dynamics in cerebral veins and microcerebral vessels caused by the pharmacologically induced acute peripheral hypertension and the sound-induced opening of BBB is performed in Sec. 3. Section 4 contains some concluding remarks.

\section{Experiments and Methods}

\subsection{Experimental procedure}

All experimental procedures were carried out in accordance with the Guide for the Care and Use of Laboratory Animals (NIH Publication No. 85-23, revised 1996). The first series of experiments was performed in mongrel normotensive male rats $(n=12)$. The animals were housed at $25 \pm 2{ }^{\circ} \mathrm{C}$, $55 \%$ humidity, and 12:12 h light/dark cycle. One day before the experimental study, animals were instrumented with catheters for the continuous recording of the mean arterial pressure (MAP). The implantation was done after the anesthesia with ketamine $(40 \mathrm{mg} / \mathrm{kg}$, ip) supplemented with xylazine $(5 \mathrm{mg} / \mathrm{kg}$, ip). The polyethylene catheter PE-50 with the PE-10 tip (Scientific Commodities INC., Lake Havasu City, Arizona) was placed in the femoral artery. Another catheter was inserted into the femoral vein for performing the phenylephrine injection leading to acute peripheral hypertension. Registration of MAP was done using the PowerLab system (ADInstruments, Australia) during $30 \mathrm{~min}$. Aiming to study how the phenylephrine-induced hypertension influences the cerebrovascular dynamics, relative CBF was measured. Recordings related to macro and microcerebral regulation were acquired for two states: (1) the base-line recording, and (2) the recording after the phenylephrine injection $(0.125 \mu \mathrm{g} / \mathrm{kg}$, iv) leading to the increased MAP by about $10 \%$. Analysis was performed for experimental data with excluded transient processes occurred after the injection. To study the phenylephrine-related responses in MAP, the segments with the strongest responses were selected.

The second series of experiments was performed in adult male mice divided into four groups: the control group and three groups of animals underwent the sound exposure, namely, $90 \mathrm{~min}, 4 \mathrm{~h}$ and $24 \mathrm{~h}$ after this exposure. Each group included 10 mice. We used the audible sound to induce the opening of BBB according to the following protocol: $60 \mathrm{~s}$ - sound $(110 \mathrm{~dB}, 370 \mathrm{~Hz})$, then $60 \mathrm{~s}$ - pause, and repeating of this cycle during $2 \mathrm{~h}$. Such type of exposure was done using the sound transducer in a Plexiglas chamber (the volume $-2000 \mathrm{~cm}^{3}$ ) that provided amplifying the effect of sound on mice. By analogy with the first series of experiments, recording of CBF-data was performed to characterize both, the venous (the sagittal sinus) and the microcerebral circulation (small cerebral vessels of the microcirculatory network).

\section{2. $\quad$ Laser speckle contrast imaging}

Measurement of CBF was done with a home-made system for LSCI. The raw speckle images were recorded by illuminating the exposed rat cortex with the HeNe laser (Thorlabs HNL210L, $632.8 \mathrm{~nm})$. The monochromatic CMOS camera Basler acA2500-14gm was supplied by imaging lens Computer M1614-MP2 $16 \mathrm{~mm}$ at $F$-number equal to 6 , that corresponds to speckle/pixel size ratio above 2 to satisfy Nyquist criteria; exposure time $-20 \mathrm{~ms}$. Speckle images were recorded with the frame rate of 40 frames/s. The contrast was estimated as $K=\sigma /\langle I\rangle$, with $\sigma$ and $\langle I\rangle$ being the standard deviation of intensity fluctuations and the mean intensity within a $5 \times 5$ sliding window, respectively. The $5 \times 5$ sliding window was chosen as a compromise between the estimation of accuracy of speckle contrast and the spatial resolution of a resulted image. ${ }^{26}$ Noise reduction was carried out by averaging within a moving window $(55 \times 55$ pixels $)$ over 50 consequent images that corresponds to time resolution $1.25 \mathrm{~s}$. Further, the averaged speckle images were converted into the flow velocity data using the Gaussian approach.

Figure 1 shows an example of speckle contrast image used for the selection of regions for the recording of CBF-data. Within each region, the position of the most probable contrast value was controlled throughout the experiment.

\subsection{Multiscale entropy}

Different entropy-based approaches are widely used to quantify transformation of normal physiological processes into the pathological dynamics. Due to the presence of many definitions of entropies, it is 


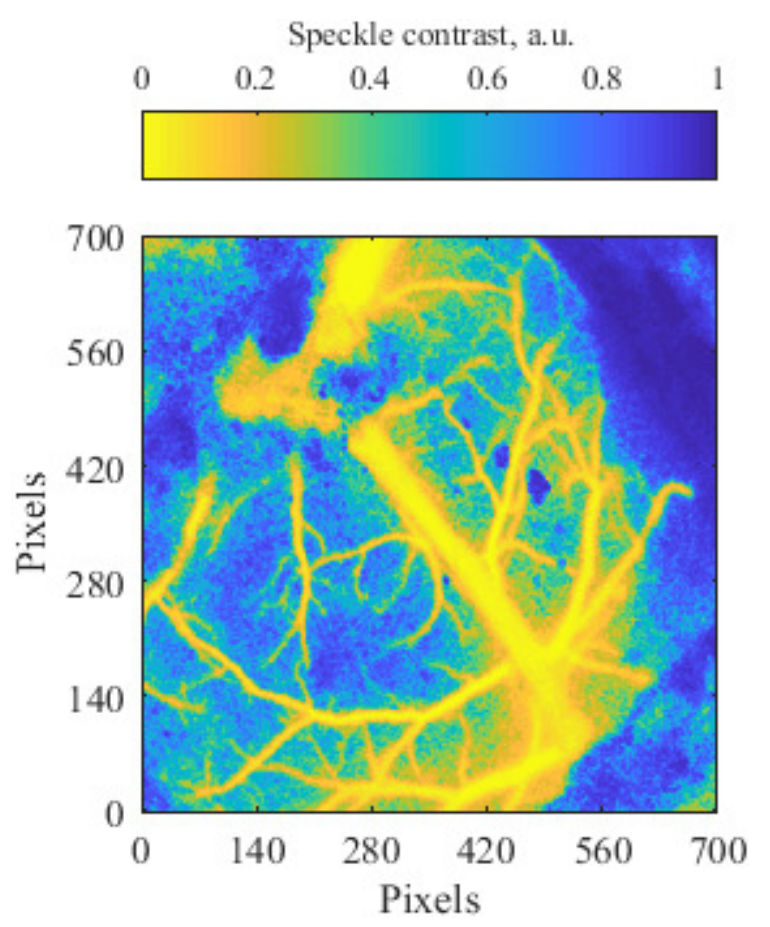

Fig. 1. An example of speckle contrast image of cerebral vessels. Here, $1 \mathrm{~mm}$ is related to 128 pixels.

not always clear how these definitions are related to each other. Traditionally, higher entropy is associated with higher complexity of data series. However, the relation between the complexity and the predictability (i.e., the regularity) of data series is not obvious. The fully predictable periodic sequence is characterized by minimal entropy value, while the white noise is quantified by maximal entropy value. Can we consider white noise as the most complex regime? It is fully unpredictable, but it is described by a single Hölder exponent, and the singularity spectrum of white noise contains a single point reflecting the monofractal structure of this random process. From such point of view, white noise is a simpler process compared with multifractal signals that require a larger set of Hölder exponents to numerically describe their structure. The absence of correlations and predictability may therefore be associated with compact representation of the analyzed process in terms of the singularity spectrum or other measures of complex scaling. Although the question such as "how to define the most complex dynamics" does not have unique answer, the intuitive definition was suggested $^{27}$ and assumed "meaningful structural richness" with the presence of correlated behavior over multiple time scales.
The MSE is a recently proposed measure that provides characterization of the complexity of physiological processes (or time series of another origin) in accordance with the mentioned intuitive definition. ${ }^{27}$ This approach outperforms entropybased measures that do not consider the multiple time scales, and provides a way of a clearer interpretation of the obtained results. Unlike other measures, there is a possibility not only to distinguish between different physiological states, but also to associate inter-state changes with the related physiological mechanisms. MSE method is based on the SampEn being a commonly used method for analysis of irregularity of time series which improves another widely used approach, the ApEn. The feature of MSE consists in consideration of different time scales with estimation of SampEn depending on the scale factor.

This method includes two steps. At the first step, coarse-grained time series $y_{j}(\tau)$ is obtained by averaging original data points $x_{i}$ within time windows that are nonoverlapped and characterized by increased length $\tau$ :

$$
y_{j}^{\tau}=\frac{1}{\tau} \sum_{i=(j-1) \tau+1}^{j \tau} x_{i}, \quad 1 \leq j \leq N / \tau .
$$

For $\tau=1$, the coarse-grained time series coincides with $x_{i}$. With increasing $\tau$, the length of $y_{j}^{\tau}$ is reduced.

At the second stage, SampEn $\left(S_{E}\right)$ is estimated for each $y_{j}^{\tau}$. This characteristic is proportional to the logarithm of the probability that two sequences of data points similar for the pattern of the length $m$ remain similar when the pattern length increases by 1 , i.e., for the length $m+1$. The definition of SampEn does not include self-matches when computing the probability. ${ }^{12,13}$

In the earlier performed MSE-based studies, ${ }^{12-14}$ a wide range of scale factor is considered, e.g., from 1 to 20 . In order to analyze such scales, the used time series should possess a sufficient length $\left(N \sim 10^{4}\right)$. When dealing with significantly shorter data series, this range needs to be reduced. In the opposite case, the available amount of data does not allow estimation of probabilities with a good precision. The coarse-graining procedure reduces the length of $y_{j}^{\tau}$, and the more is $N$, the higher scaling factors can be considered. For data series of about $N \sim 300$ samples which are taken in this study, we used scale factors in the range from 1 to 5 that 
correspond to time scales $1.25,2.5,5,10$ and $20 \mathrm{~s}$. The MSE-approach can be applied to such small amount of data resulting in larger SD of the estimated quantities. ${ }^{12}$ Nevertheless, this approach may distinguish between normal and pathological states if the corresponding differences exceed the intra-group variations.

In this study, we used the software for computing MSE developed by M. Costa (http://www.physionet.org). ${ }^{28}$

\section{Results and Discussion}

In this study, we used the MSE approach for distinguishing CBF-dynamics associated with short-term phenylephrine-related acute peripheral hypertension from the base-line measurements. Because the response of cerebral dynamics to the injection of phenylephrine is nonstationary (the strongest reaction takes few minutes and then it is slowly reduced), we performed a selection of fragments of the CBF-velocity data consisting of about $N=350-400$ samples. In order to estimate MSE, the values of two algorithmic parameters should be specified, namely, the pattern length $m$ and the similarity criterion $r$. The illustrated results are obtained for the default parameter values $(m=2$, $r=0.15)$.

Figure 2 shows the estimated entropy depending on the scale factor for CBF velocity data related to macroscopic dynamics in sagittal sinus (Fig. 2(a)) and the surrounding network of small cerebral

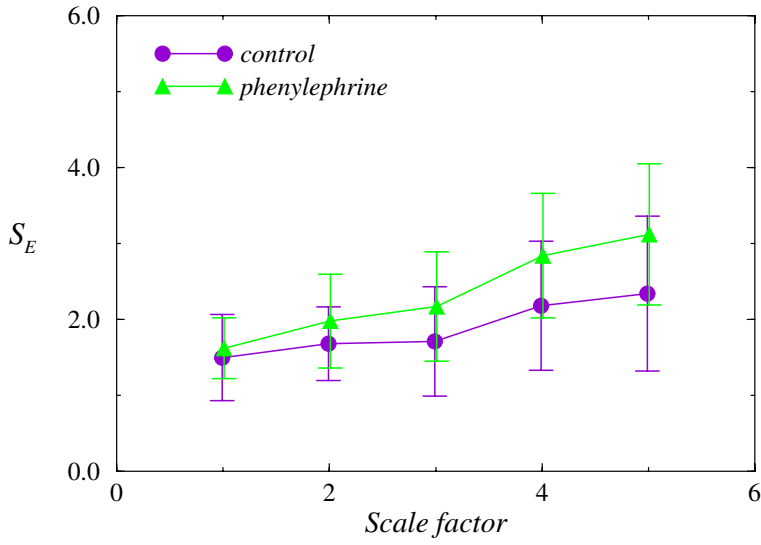

(a) vessels (Fig. 2(b)). For the case of macroscopic cerebral circulation, the Mann-Whitney test confirms the absence of significant distinctions $(p>0.05)$ in the CBF-dynamics after the phenylephrine injection compared with the control group. A small increase of SampEn $S_{E}$ is observed that is more pronounced for large-scale factor. However, the inter-group distinctions are not enough to separate the considered physiological states as compared with intra-group variations. We can conclude therefore that macroscopic CBF-dynamics does not allow revealing the effects of phenylephrine-related acute peripheral hypertension, at least for short recordings of $\mathrm{CBF}$-velocity data.

The microcerebral circulation associated with the blood flow dynamics in small cerebral vessels surrounding the sagittal sinus is more sensitive to abrupt changes in the peripheral arterial pressure. The inter-group differences shown in Fig. 2(b) are essentially larger than the corresponding distinctions observed for macroscopic cerebral vessels (Fig. 2(a)). They are quantified as significant changes by the Mann-Whitney test $(p<0.05)$ for large-scale factor. Now, the inter-group distinctions outperform the intra-group variations confirming that the dynamics of small cerebral vessels shows a response to the acute peripheral hypertension. This response can be diagnosed based on short fragments of experimental recordings. The latter conclusion is in accordance with other studies ${ }^{29}$ that demonstrate a higher sensitivity of microcerebral dynamics to the peripheral arterial pressure. Following the traditional physiological assumptions,

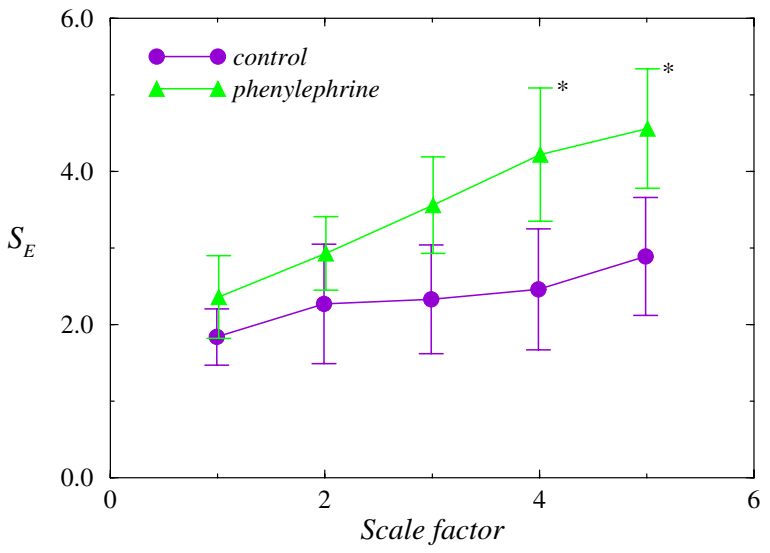

(b)

Fig. 2. MSE-analysis of (a) macrocerebral and (b) microcerebral CBF-dynamics related to phenylephrine-induced acute peripheral hypertension and base-line measurement in the control group. Stars are related to significant inter-group distinctions. Here, $p \simeq 0.01 \div 0.02$. 


\section{A. N. Pavlov et al.}

the model of static cerebral autoregulation is mainly considered $^{30}$ that is verified by the independence of $\mathrm{CBF}$ when the arterial blood pressure is varied in a wide range (e.g., $60-150 \mathrm{~mm} \mathrm{Hg}$ in humans). On the contrary, recent studies suggested a relation between a high risk for the stroke caused by pathological changes in the mechanisms regulating the CBF-dynamics and the systemic hypertension. ${ }^{31-33}$ The results of this paper confirm that the peripheral pressure variations provide a response in cerebral circulation and, therefore, the model of static cerebral autoregulation does not account for the vessels dynamics in the microcirculatory network.

Further, we considered changes in the $\mathrm{CBF}$ associated with the intermittent sound. Sound exposure may influence the BBB permeability. Pioneering ex vivo studies performed in 1940th years $^{34,35}$ suggested that cerebral microvessels represent the anatomic site of the BBB. Significant progress of in vivo analysis of cerebral dynamics provided the ability of in vivo study of BBB physiology, and the LSCI represents an appropriate tool that can be used for this purpose. In the previous paper, ${ }^{36}$ we have shown that the BBB opens in $90 \mathrm{~min}$ after the sound exposure that leads to suppressed cerebral venous circulation. Further, the BBB permeability normalizes (about $4 \mathrm{~h}$ after the sound exposure), however, the prolonged accumulation of water in the brain parenchyma due to the long-lasting processes of recovery from vasogenic edema, probably, provokes increase in the intracranial pressure which induces once again BBB opening. ${ }^{36}$ The latter process is rather weak as

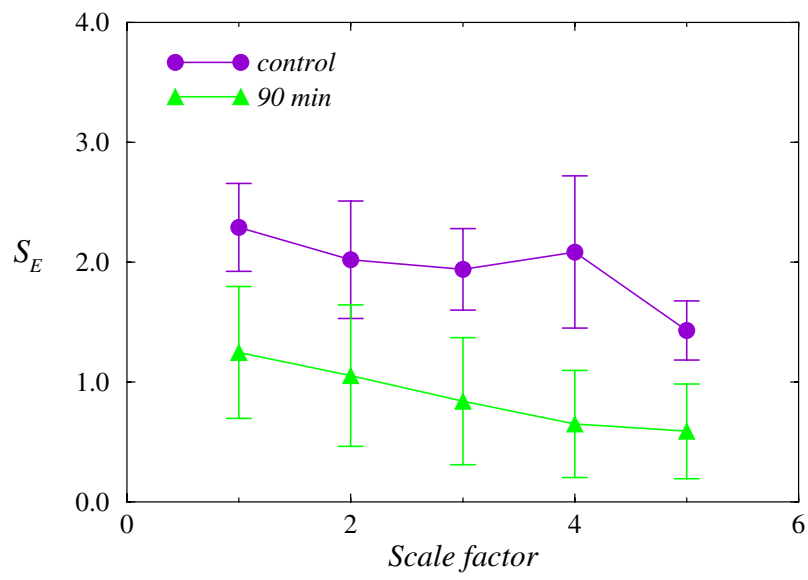

(a) compared with changes in the venous CBF that occur in $90 \mathrm{~min}$ after the sound exposure.

Let us consider how these changes can be quantified by the MSE-method. Analysis of the CBF-data was provided by analogy with the first series of experiments with the same parameters of MSE-method. According to Fig. 3(a), strong changes of SampEn related to BBB opening are observed in CBF-dynamics in the cerebral veins. They occur in the whole range of the considered scale factor ( $p<0.05$ according to the Mann-Whitney test). Depending on the scale factor, these distinctions can be made clearer (see, e.g., scale factor 4 in Fig. 3(a)). We would like to note that significant distinctions outperforming the intra-group variations are observed for quite short data series verifying the method's abilities of quantifying changes in CBF-dynamics for a very limited amount of experimental data.

In the case of microcerebral circulation, we do not observe analogous changes (Fig. 3(b)). Independently on the scale factor, the inter-group distinctions are fairly insignificant relative to variations of characteristics for animals within each group. Thus, the consideration of CBF-velocity in small cerebral vessels of microcirculatory network does not enable characterizing changes caused by the BBB opening. The latter may be explained by the presence of compensatory mechanisms that provide distribution of blood in the cerebral veins as capacity link of the CBF.

Figure 4 shows how the SampEn changes depending on the time duration after the sound

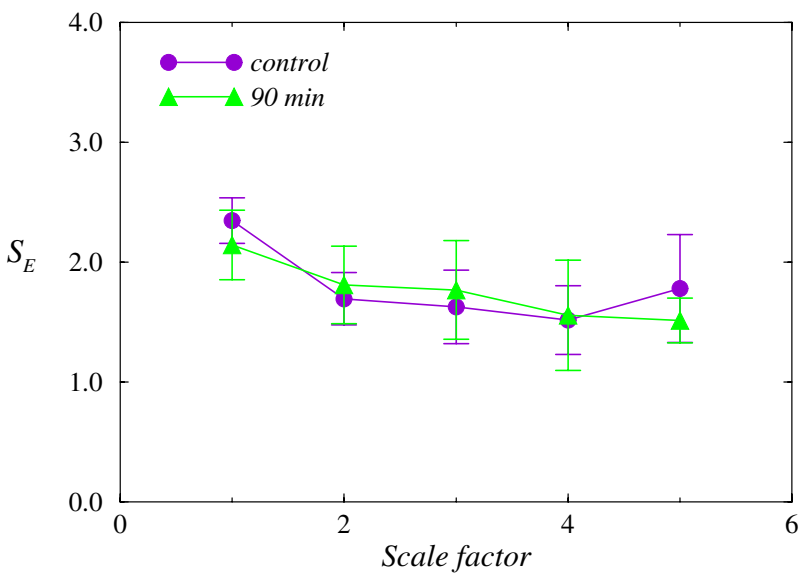

(b)

Fig. 3. MSE-analysis of (a) macrocerebral and (b) microcerebral CBF-dynamics related to the control group and to the group of mice with sound exposure. 


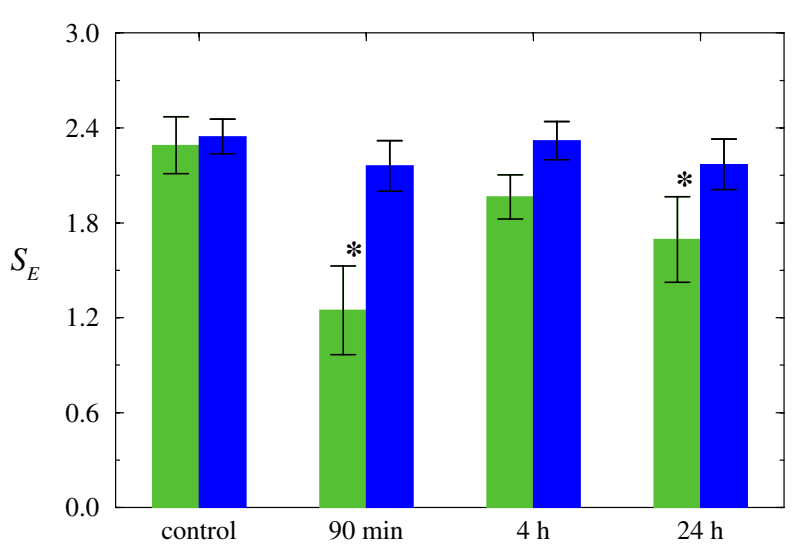

Fig. 4. Statistical analysis of macrocerebral (green boxes) and microcerebral (blue boxes) dynamics of different physiological states. Stars are related to significant inter-group distinctions $(p \simeq 0.006$ for $90 \mathrm{~min}, p \simeq 0.015$ for $24 \mathrm{~h}$ ).

exposure (the results are shown for the scale factor 4). At the level of macrocerebral circulation, strong changes in $90 \mathrm{~min}$ become smaller in $4 \mathrm{~h}$ after the sound exposure when the $S_{E}$ measure approaches to the estimations performed in the control group. Inter-group distinctions (compared with the control group) increase in $24 \mathrm{~h}$, but they are smaller than in $90 \mathrm{~min}$. At the level of microcerebral circulation, no essential changes occur during the whole experimental study. Visually, some distinctions are observed in $90 \mathrm{~min}$ and in $24 \mathrm{~h}$ as compared with the control group, however, $p$-value slightly increases to 0.05 .

Note, that other entropy-based measures can also provide inter-group distinctions for macrocerebral circulation. In particular, the Shannon entropy has showed differences between the control group and the group of animals analyzed in $90 \mathrm{~min}$ after the sound exposure. However, the $p$-value was about two times higher, and the obtained results do not allow to establish a relation between changes in the complexity and the mechanisms responsible for these changes. With MSE, we obtain more information. Thus, the strongest changes were observed for the scale factors $4-5$ that correspond to the time scales about $10-20 \mathrm{~s}$. The latter allows to associate the observed changes with the NO-related endothelial function.

\section{Conclusions}

In this work, we discussed how the entropybased analysis allows characterization of the cerebrovascular dynamics in large and small blood vessels and reported its changes caused by the acute peripheral hypertension and the sound-induced BBB opening. Due to complex organization of CBF-velocity data with multiple scales reflecting the impact of distinct regulatory mechanisms, application of measures that quantify the whole cerebrovascular dynamics within a single quantity seems to be a less informative approach compared with the scale-related analysis. Despite the presence of many complexity measures, here we applied the MSE-technique that can be used in the case of adaptive dynamics of living systems characterized by the clearly expressed multiscality.

We showed that the phenylephrine-related increase in the arterial pressure has a response in the microcirculatory network of cerebral vessels that is quantified by larger values of SampEn compared with the base-line measurements. These changes are scale-dependent and they are more essential at larger-scale factor. CBF-dynamics in macrocerebral vessels does not show pronounced distinctions between the control group and the group of rats with acute peripheral hypertension. Possible inter-group differences do not exceed the intra-group variability, thus demonstrating the absence of authentic separation when processing quite short data series considered in this study. An increased amount of data may reduce the values of SD of SampEn estimated for both groups, however, a short-term reaction caused by the injection of phenylephrine does not allow considering essentially larger data series. Thus, the microcerebral circulation is significantly more sensitive to abrupt changes in the peripheral arterial pressure as compared with macrocerebral dynamics.

Additionally, we demonstrated the ability of characterizing the $\mathrm{BBB}$ permeability with the MSE-method. In the analysis of experimental data of $\mathrm{CBF}$ velocity related to different stages of soundinduced changes in the cerebral vessels functioning, we again revealed distinctions between the macroand microcerebral dynamics. Blood flow dynamics in cerebral veins shows strong changes of MSE that occur during the BBB opening, and these changes are mainly observed in $90 \mathrm{~min}$ after the end of the sound exposure. At the level of microcerebral vessels, no observable distinctions occur throughout the whole experiment. We assume that such distinctions between macro and microcerebral circulation may be caused by compensatory mechanisms 
providing blood distribution in the cerebral veins being the capacity link of the CBF.

\section{Acknowledgment}

This work was supported by the Russian Science Foundation (Agreement No. 17-15-01263).

\section{References}

1. C. E. Shannon, "A mathematical theory of communication," Bell Sys. Tech. J. 27, 379-423 (1948).

2. C. Bandt, B. Pompe, "Permutation entropy: A natural complexity measure for time series," Phys. Rev. Lett. 88, 174102 (2002).

3. S. M. Pincus, "Approximate entropy as a measure of system complexity," Proc. Natl. Acad. Sci. 88, 2297-2301 (1991).

4. J. S. Richman, J. R. Moorman, "Physiological timeseries analysis using approximate entropy and sample entropy," Am. J. Physiol. Heart Circ. Physiol. 278, H2039-H2049 (2000).

5. A. L. Goldberger, C. K. Peng, L. A. Lipsitz, "What is physiologic complexity and how does it change with aging and disease?" Neurobiol. Aging 23, 23-26 (2002).

6. L. A. Lipsitz, A. L. Goldberger, "Loss of 'complexity' and aging. Potential applications of fractals and chaos theory to senescence," J. Am. Med. Assoc. 267, 1806-1809 (1992).

7. M. P. Tulppo, A. M. Kiviniemi, A. J. Hautala, M. Kallio, T. Seppönen, T. H. Mökikallio, H. V. Huikuri, "Physiological background of the loss of fractal heart rate dynamics," Circulation 112, 314319 (2005).

8. A. N. Pavlov, O. N. Pavlova, O. V. Sosnovtseva, E. Mosekilde, N.-H. Holstein-Rathlou, "Characterizing multimode interaction in renal autoregulation," Physiol. Meas. 29, 945-958 (2008).

9. A. N. Pavlov, O. V. Semyachkina-Glushkovskaya, O. N. Pavlova, A. S. Abdurashitov, G. M. Shihalov, E. V. Rybalova, S. S. Sindeev, "Multifractality in cerebrovascular dynamics: An approach for mechanisms-related analysis," Chaos Solitons Fractals 91, 210-213 (2016).

10. S. M. Pincus, "Assessing serial irregularity and its implications for health," Ann. N. Y. Acad. Sci. 954, 245-267 (2002).

11. D. E. Lake, J. S. Richman, M. P. Griffin, J. R. Moorman, "Sample entropy analysis of neonatal heart rate variability," Am. J. Physiol. Regul. Integr. Comp. Physiol. 283, R789-R797 (2002).
12. M. Costa, A. L. Goldberger, C.-K. Peng, "Multiscale entropy analysis of biological signals," Phys. Rev. E 71, 021906 (2005).

13. M. Costa, A. L. Goldberger, C.-K. Peng, "Multiscale entropy analysis of physiologic time series," Phys. Rev. Lett. 89, 062102 (2002).

14. M. Costa, C.-K. Peng, A. L. Goldberger, J. M. Hausdorff, "Multiscale entropy analysis of human gait dynamics," Physica A 330, 53-60 (2003).

15. J. D. Briers, S. Webster, "Laser speckle contrast analysis (LASCA): A nonscanning, full-field technique for monitoring capillary blood flow," J. Biomed. Opt. 1, 174-179 (1996).

16. D. A. Boas, A. K. Dunn, "Laser speckle contrast imaging in biomedical optics," J. Biomed. Opt. 15, 011109 (2010).

17. A. N. Pavlov, O. N. Pavlova, V. V. Tuchin, O. V. Semyachkina-Glushkovskaya, Y. Zhang, O. A. Bibikova, Q. Huang, D. Zhu, P. Li, Q. Luo, "Multiresolution analysis of pathological changes in cerebral venous dynamics in newborn mice with intracranial hemorrhage: Adrenorelated vasorelaxation," Physiol. Meas. 35, 1983-1999 (2014).

18. A. K. Dunn, "Laser speckle contrast imaging of cerebral blood flow," Ann. Biomed. Eng. 40, 367377 (2012).

19. G. A. Armitage, K. G. Todd, A. Shuaib, I. R. Winship, "Laser speckle contrast imaging of collateral blood flow during acute ischemic stroke," Cereb. Blood Flow Metab. 30, 1432-1436 (2010).

20. A. J. Strong, E. L. Bezzina, P. J. Anderson, M. G. Boutelle, S. E. Hopwood, A. K. Dunn, "Evaluation of laser speckle flowmetry for imaging cortical perfusion in experimental stroke studies: Quantitation of perfusion and detection of peri-infarct depolarisations," J. Cereb. Blood Flow Metab. 26, 645-653 (2006).

21. N. Feng, J. Qiu, P. Li, X. Sun, C. Yin, W. Luo, S. Chen, Q. Luo, "Simultaneous automatic arteriesveins separation and cerebral blood flow imaging with single-wavelength laser speckle imaging," Opt. Express 19, 15777-15791 (2011).

22. A. S. Abdurashitov, V. V. Lychagov, O. A. Sindeeva, O. V. Semyachkina-Glushkovskaya, V. V. Tuchin, "Histogram analysis of laser speckle contrast image for cerebral blood flow monitoring," Front. Optoelectron. 8, 187-194 (2015).

23. A. N. Pavlov, A. I. Nazimov, O. N. Pavlova, V. V. Lychagov, V. V. Tuchin, O. A. Bibikova, S. S. Sindeev, O. V. Semyachkina-Glushkovskaya, "Waveletbased analysis of cerebrovascular dynamics in newborn rats with intracranial hemorrhages," $J$. Innov. Opt. Health Sci. 7, 1350055 (2014).

24. O. Semyachkina-Glushkovskaya, A. Pavlov, J. Kurths, E. Borisova, A. Gisbrecht, O. Sindeeva, 
A. Abdurashitov, A. Shirokov, N. Navolokin, E. Zinchenko, A. Gekalyuk, M. Ulanova, D. Zhu, Q. Luo, V. Tuchin, "Optical monitoring of stressrelated changes in the brain tissues and vessels associated with hemorrhagic stroke in newborn rats," Biomed. Opt. Express 6, 4088-4097 (2015).

25. O. V. Semyachkina-Glushkovskaya, V. V. Lychagov, A. S. Abdurashitov, O. V. Sindeeva, S. S. Sindeev, E. M. Zinchenko, E. I. Kajbeleva, A. N. Pavlov, M. Kassim, V. V. Tuchin, "Changes in the cerebral blood flow in newborn rats assessed by LSCI and DOCT before and after the hemorrhagic stroke," Proc. SPIE 9305, 93051D (2015).

26. D. D. Duncan, S. J. Kirkpatrick, R. K. Wang, "Statistics of local speckle contrast," J. Opt. Soc. Am. A 25, 9-15 (2008).

27. P. Grassberger, Information and complexity measures in dynamical system, Information Dynamics, by H. Atmanspacher, H. Scheingraber, Eds., pp. 15-33, Plenum Press, New York (1991).

28. A. L. Goldberger, L. A. Nunes Amaral, L. Glass, J. M. Hausdorff, P. Ch. Ivanov, R. G. Mark, J. E. Mietus, G. B. Moody, C.-K. Peng, H. E. Stanley, "PhysioBank, PhysioToolkit, and PhysioNet: Components of a new research resource for complex physiologic signals," Circulation 101(23), e215-e220 (2000), Circulation Electronic Pages; http://circ.ahajournals.org/cgi/content/full/101/ 23/e215.

29. A. N. Pavlov, A. S. Abdurashitov, O. A. Sindeeva, S. S. Sindeev, O. N. Pavlova, G. M. Shihalov, O. V. Semyachkina-Glushkovskaya, "Characterizing cerebrovascular dynamics with the wavelet-based multifractal formalism," Physica A 442, 149-155 (2016).

30. N. A. Lassen, "Cerebral blood flow and oxygen consumption in man," Physiol. Rev. 39, 183-238 (1959).

31. L. Beason-Held, A. Moghekar, A. Zonderman, M. Kraut, S. M. Resnick, "Longitudinal changes in cerebral blood flow in the older hypertensive brain," Stroke 38, 1766-1773 (2007).

32. P. J. Gianaros, S. W. Derbyshire, J. C. May, G. J. Siegle, M. A. Gamalo, J. R. Jennings, "Anterior cingulate activity correlates with blood pressure during stress," Psychophysiology 42(6), 627-635 (2005).

33. P. J. Gianaros, P. J. Greer, C. M. Ryan, J. R. Jennings, "Higher blood pressure predicts lower regional gray matter volume: Consequences on short-term information processing," Neuroimage 31(2), 754-765 (2006).

34. T. Broman, "The possibilities of the passage of substances from the blood to the central nervous system," Acta Psychiat. Scacd. 16, 1-25 (1941).

35. U. Friedemann, "Blood-brain barrier," Physiol. Rev. 22, 125-142 (1942).

36. O. Semyachkina-Glushkovskaya, A. Abdurashitov, A. Pavlov, A. Shirokov, N. Navolokin, O. Pavlova, A. Gekalyuk, M. Ulanova, N. Shushunova, A. Bodrova, E. Saranceva, A. Khorovodov, I. Agranovich, V. Fedorova, M. Sagatova, A. E. Shareef, C. Zhang, D. Zhu, V. Tuchin, "Laser speckle imaging and wavelet analysis of cerebral blood flow associated with the opening of blood-brain barrier by sound," Chin. Opt. Lett. 15(9), 090002 (2017). 